\title{
Kinetics and mechanism of the oxidation of some $\alpha$-hydroxy acids by hexamethylenetetramine-bromine
}

\author{
DIMPLE GARG and SEEMA KOTHARI* \\ Department of Chemistry, J N V University, Jodhpur 342 005, India \\ e-mail: seema@goraexports.com; skothari23@rediffmail.com
}

MS received 3 June 2004; revised 8 November 2004

\begin{abstract}
The oxidation of lactic acid, mandelic acid and ten monosubstituted mandelic acids by hexamethylenetetramine-bromine (HABR) in glacial acetic acid, leads to the formation of the corresponding oxoacid. The reaction is first order with respect to each of the hydroxy acids and HABR. It is proposed that HABR itself is the reactive oxidizing species. The oxidation of $\alpha$-deuteriomandelic acid exhibits the presence of a substantial kinetic isotope effect $\left(k_{H} / k_{D}=5.91\right.$ at $\left.298 \mathrm{~K}\right)$. The rates of oxidation of the substituted mandelic acids show excellent correlation with Brown's $\sigma^{+}$values. The reaction constants are negative. The oxidation exhibits an extensive cross conjugation between the electron-donating substituent and the reaction centre in the transition state. A mechanism involving transfer of a hydride ion from the acid to the oxidant is postulated.
\end{abstract}

Keywords. Kinetics; oxidation; $\alpha$-hydroxy acids; hexamethyleneteramine-bromine.

\section{Introduction}

Hexamethylenetetramine-bromine (HABR) was first reported as a synthetic reagent for the oxidation of alcohols to carbonyl compounds in $1994 .{ }^{1}$ HABR is a non-hygroscopic solid, easily handled and storable. It is not affected by ordinary exposure to light, air or water and has no offensive odour of bromine. Hence, it is a safe and convenient source of active bromine in comparison to bromine which is a highly toxic oxidizer. We have undertaken a study of the kinetics and mechanism of oxidations by HABR and some reports have emanated from our laboratory on the mechanistic aspects of oxidations by HABR. ${ }^{2-5}$ A perusal of literature showed that the kinetics and mechanism of the oxidation of hydroxy acids by HABR has not been investigated so far. Hydroxy acids may be oxidized either as alcohols yielding corresponding oxoacids ${ }^{6}$ or may undergo decarboxylation to yield an aldehyde. ${ }^{7}$ In the present work, the kinetics of oxidation by HABR, in glacial acetic acid, of lactic acid (LA), mandelic acid (MA) and ten substituted mandelic acids have been described. Attempts have been made to correlate rate and structure in this reaction. Mechanistic aspects are discussed.

\footnotetext{
*For correspondence
}

\section{Experimental}

\subsection{Materials}

HABR was prepared by the reported method ${ }^{1}$ and its purity checked by an iodometric method and melting point determination. Contrary to the earlier report, ${ }^{1}$ we found that the active bromine content of this complex is 2 mol per mole of the reagent. LA, MA and nitromandelic acids used were commercial products of the highest purity available and were used as such. Halogeno-, alkyl- and $p$-methoxymandelic acids were prepared from the corresponding benzaldehydes by cyanohydrine formation followed by acid hydrolysis. ${ }^{8}$ Solutions were standardized by alkalimetry. $\alpha$-Deuteriomandelic acid (DMA) was prepared by the method of Kemp and Waters, ${ }^{9}$ and its isotopic purity, as ascertained by its NMR spectrum was $94 \pm 5 \%$. Glacial acetic acid was purified by refluxing it with $\mathrm{CrO}_{3}$ and acetic anhydride for $6 \mathrm{~h}$ and then distilled. ${ }^{10}$

\subsection{Product analysis}

The formation of oxoacids as the product of the oxidation was confirmed by its characteristic positive spot test, given by a completely reduced reaction mixture. ${ }^{11}$ The quantitative estimation of oxoacids were carried out under kinetic conditions. In a typical experiment, 
mandelic acid $(0.1 \mathrm{~mol})$ and HABR $(0.01 \mathrm{~mol})$ were dissolved in $100 \mathrm{ml}$ glacial acetic acid. The reaction mixture was allowed to stand in the dark for $\approx 6 \mathrm{~h}$ to ensure completion of the reaction. Most of the solvent was removed by distillation under reduced pressure. The solution was then treated with excess $(250 \mathrm{ml})$ of a saturated solution of 2,4-dinitrophenylhydrazine in $2 \mathrm{~mol} \mathrm{dm}^{-3} \mathrm{HCl}$ and kept overnight in a refrigerator. The precipitated 2,4-dinitrophenylhydrazone (DNP) was filtered off, dried, weighed, recrystallized from ethanol, and weighed again. The yields of DNP before and after recrystallization was $2.22 \mathrm{~g}$ $(91 \%)$ and $2.03 \mathrm{~g}(83 \%)$ respectively. The identity of product was established by comparing the m.p. of the DNP derivative with the literature value. ${ }^{12}$ The DNP was found identical (m.p. $=192^{\circ} \mathrm{C}$ and mixed m.p. $=194^{\circ} \mathrm{C}$ ) with the DNP of phenylglyoxylic acid $\left(\mathrm{m} . \mathrm{p} .=196-7^{\circ} \mathrm{C}\right) .{ }^{12}$ In similar experiments, with other hydroxy acids, the yield of the corresponding oxoacids was in the range of $77-87 \%$, after recrystallization.

\subsection{Stoichiometry}

To determine the stoichiometry, HABR $(0.05 \mathrm{~mol})$ and MA $(0.01 \mathrm{~mol})$ were made up to $100 \mathrm{ml}$ in glacial acetic acid. The reaction was allowed to stand for $\approx 10 \mathrm{~h}$ to ensure the completion of the reaction. The residual HABR was determined iodometrically. Several determinations, with different concentrations of MA, showed that the stoichiometry is $2: 1$ i.e. two moles of hydroxy acids are oxidized per mole of HABR.

\subsection{Spectral studies}

UV-Vis spectra of HABR, hexamethylenetetramine (HXA) and bromine were obtained, on a HP diodearray rapid scanning spectrophotometer (Model 8452A) with a scanning speed of $600 \mathrm{~nm} \mathrm{~s}^{-1}$. The solvent was glacial acetic acid and temperature was $\approx 298 \mathrm{~K}$.

\subsection{Kinetic measurements}

The reactions were studied under pseudo first-order conditions by maintaining an excess $(\times 20$ or greater) of the hydroxy acid over HABR, solvent glacial acetic acid. The reactions were carried out at constant temperature $( \pm 0 \cdot 1 \mathrm{~K})$ and were followed iodometrically by monitoring the decrease in [HABR] for up to more than $80 \%$ of the reaction. Pseudo first- order rate constants, $k_{\mathrm{obs}}$, were evaluated from linear plots $\left(c^{2}>0.995\right)$ of $\log$ [HABR] against time. Duplicate kinetic runs showed that the rates were reproducible to within $\pm 4 \%$. The second order rate constant, $k_{2}$, was calculated by the following relation: $k_{2}=k_{\mathrm{obs}} /$ [hydroxy acid]. We have used coefficient of determination $\left(c^{2}\right)$, standard deviation (sd) and Exner's ${ }^{13}$ parameter $\psi$, as the measures of goodness of fit in correlation analysis.

\section{Results}

Rates and other experimental data were obtained for all the hydroxy acids. Since the results are similar, only representative data are reproduced here.

Oxidation of the hydroxy acids by HABR results in the formation of the corresponding oxoacids as individual compounds. Analyses of product and stoichiometric determinations indicate the following overall reaction.

$$
\begin{aligned}
& 2 \mathrm{RCH}(\mathrm{OH}) \mathrm{COOH}+\left(\mathrm{CH}_{2}\right)_{6} \mathrm{~N}_{4} \mathrm{Br}_{4} \rightarrow \\
& \mathrm{RCOCOOH}+\left(\mathrm{CH}_{2}\right)_{6} \mathrm{~N}_{4}+4 \mathrm{HBr}
\end{aligned}
$$

\subsection{Rate laws}

The reactions are first order with respect to HABR as evidenced by a linear plot of log [HABR] time. The pseudo first-order rate constants do not depend on the initial concentration of HABR (table 1). This further confirms first-order dependence of rate on oxidant concentration. Reaction rates increase linearly with increase in the concentrations of the hydroxy acid (table 1). A plot of $\log k_{\text {obs }}$ vs log [hydoxy acid] is linear $\left(c^{2}>0.999\right)$ with a slope $=0.97 \pm 0.02$, indicating that the order with respect to hydroxy acid is also one.

\subsection{Induced polymerization of acrylonitrile}

Oxidation of hydroxy acids, under nitrogen atmosphere, failed to induce polymerization of acrylonitrile. Further, addition of acrylonitrile had no effect on the rate (table 1).

\subsection{Effect of temperature}

Rates of oxidation of hydroxy acids were determined at different temperatures between 298 and $328 \mathrm{~K}$ and the activation parameters were calculated (table 2). 
Table 1. Rate constants for the oxidation of mandelic acid by HABR at $318 \mathrm{~K}$.

\begin{tabular}{|c|c|c|}
\hline $\begin{array}{l}{[\mathrm{MA}]} \\
\left(\mathrm{mol} \mathrm{dm}{ }^{-3}\right)\end{array}$ & $\begin{array}{c}10^{3}[\mathrm{HABR}] \\
\left(\mathrm{mol} \mathrm{dm}^{-3}\right)\end{array}$ & $\begin{array}{c}10^{4} k_{\text {obs }} \\
\left(\mathrm{s}^{-1}\right)\end{array}$ \\
\hline $0 \cdot 2$ & $1 \cdot 0$ & $1 \cdot 20$ \\
\hline 0.4 & $1 \cdot 0$ & $2 \cdot 30$ \\
\hline $0 \cdot 6$ & $1 \cdot 0$ & $3 \cdot 15$ \\
\hline $1 \cdot 0$ & $1 \cdot 0$ & $5 \cdot 40$ \\
\hline $2 \cdot 0$ & $1 \cdot 0$ & $11 \cdot 2$ \\
\hline $3 \cdot 0$ & $1 \cdot 0$ & $16 \cdot 0$ \\
\hline $5 \cdot 0$ & 1.0 & $27 \cdot 5$ \\
\hline 3.0 & 1.0 & $15 \cdot 8^{\mathrm{a}}$ \\
\hline $1 \cdot 0$ & $0 \cdot 4$ & $5 \cdot 37$ \\
\hline $1 \cdot 0$ & 0.8 & $5 \cdot 45$ \\
\hline $1 \cdot 0$ & $2 \cdot 0$ & $5 \cdot 31$ \\
\hline 1.0 & $3 \cdot 0$ & $5 \cdot 51$ \\
\hline $1 \cdot 0$ & $4 \cdot 0$ & $5 \cdot 40$ \\
\hline 1.0 & $1 \cdot 0$ & $5 \cdot 48^{b}$ \\
\hline 1.0 & 1.0 & $5 \cdot 37^{\mathrm{c}}$ \\
\hline
\end{tabular}

\subsection{Kinetic isotope effect}

To ascertain the importance of the cleavage of the $\alpha-\mathrm{C}-\mathrm{H}$ bond in the rate-determining step, the oxidation of DMA was studied. The results showed (table 2 ) that the reaction exhibited a substantial kinetic isotope effect $\left(k_{\mathrm{H}} / k_{\mathrm{D}}=5.91\right.$ at $\left.298 \mathrm{~K}\right)$.

\subsection{Effect of hexamethylenetetramine}

An addition of hexamethylenetetramine (HXA) showed no effect on the rate of oxidation (table 1).

\subsection{Effect of bromide ion}

The rate of oxidation of the hydroxy acids was not affected by the addition of sodium bromide (table 1).

\subsection{Spectral studies}

Study of the UV-Vis spectra of HABR $\left(0.001 \mathrm{~mol} \mathrm{dm}^{-3}\right)$ and an equivalent amount of bromine $\left(0.002 \mathrm{~mol} \mathrm{dm}^{-3}\right)$ in glacial acetic acid at $\approx 298 \mathrm{~K}$, showed that there is some difference in the nature of the spectra of HABR and bromine. There is a broad peak for HABR in the range of $300-310 \mathrm{~nm}$ and a peak at $278 \mathrm{~nm}$ for bromine. Their optical densities showed large variations (figure 1). Hexamethylenetetramine (HXA) had no appreciable absorption in this range. Further, the spe- ctrum of HABR did not show any change in experimental time period $(\approx 2 \mathrm{~h})$. When a solution of HABR in glacial acetic acid was evaporated to dryness under reduced pressure, HABR was recovered unchanged. This confirmed that HABR retained its integrity in acetic acid.

\section{Discussion}

The linear correlation between $\log k_{2}$ of twelve hydroxy acids at 298 and $328 \mathrm{~K}\left(c^{2}=0.9994\right.$; slope $=0.857 \pm$ 0.002 ) shows that an isokinetic relationship exists in the oxidation of hydroxy acids by HABR. ${ }^{14}$ The value of the isokinetic temperature is $834 \pm 28 \mathrm{~K}$. Isokinetic relationship is a necessary condition for the validity of linear free energy relationships. It also implies that all the hydroxy acids so correlated are oxidized by similar mechanisms. ${ }^{14}$

In the present reaction, HABR itself is suggested as the reactive oxidising species from the spectral evidence and the isolation of unchanged HABR when a solution of HABR in glacial acetic acid is evaporated to dryness. Similar reactive oxidising species are suggested in the oxidation of organic sulphides by HABR. ${ }^{3}$ The nil effect of added HXA or bromide ion on the rate of oxidation is reminiscent of the results obtained earlier ${ }^{3}$ and indicates that the following equilibrium (2) does not exist in the solution.

$$
\left(\mathrm{CH}_{2}\right)_{6} \mathrm{~N}_{4} \mathrm{Br}_{4} \rightleftharpoons 2 \mathrm{Br}_{2}+\left(\mathrm{CH}_{2}\right)_{6} \mathrm{~N}_{4} .
$$

Nothing much is known about the structure of HABR. For the sake of convenience, we have shown one of the two reactive portions as $\supsetneq \mathrm{NL} \mathrm{BrL} \mathrm{Br}{ }^{5}$ For the nature of the bonding, it can be suggested that there is an interaction between the non-bonded pair of electrons on nitrogen and bromine represented by dotted lines.

It is good to mention here that the use of HABR in an acid medium has advantages over the use of bromine because the kinetics of oxidation by bromine in acid medium $(\mathrm{pH} \leq 5)$ is complex. ${ }^{15}$ In this system the probable oxidizing species may be $\mathrm{Br}_{2}$, $\mathrm{HOBR}$ or $\mathrm{Br}_{3}^{-}$. However, such complications do not arise with HABR as it retains its integrity in acetic acid.

\subsection{Correlation analysis of reactivity}

Rate constants of the oxidation of MA and ten monosubstituted mandelic acids were correlated in terms 
Table 2. Rate constants and activation parameters of the oxidation of substituted mandelic acids by $\mathrm{HABR}^{\mathrm{a}}$.

\begin{tabular}{|c|c|c|c|c|c|c|c|}
\hline \multirow[b]{2}{*}{ Substitutent } & \multicolumn{4}{|c|}{$10^{4} k_{2}\left(\mathrm{dm}^{3} \mathrm{~mol}^{-1} \mathrm{~s}^{-1}\right)$ at temp. $(\mathrm{K})$} & \multirow{2}{*}{$\begin{array}{c}\Delta H^{*} \\
\left(\mathrm{~kJ} \mathrm{~mol}^{-1}\right)\end{array}$} & \multirow{2}{*}{$\begin{array}{c}\Delta S^{*} \\
\left(\mathrm{~J} \mathrm{~mol}^{-1} \mathrm{~K}^{-1}\right)\end{array}$} & \multirow{2}{*}{$\begin{array}{c}\Delta G^{*} \\
\left(\mathrm{~kJ} \mathrm{~mol}^{-1}\right)\end{array}$} \\
\hline & 298 & 308 & 318 & 328 & & & \\
\hline $\mathrm{H}$ & $1 \cdot 30$ & $2 \cdot 71$ & $5 \cdot 40$ & $11 \cdot 0$ & $55 \cdot 1 \pm 0.6$ & $-135 \pm 2$ & $95 \cdot 2 \pm 0.5$ \\
\hline$p-\mathrm{F}$ & 1.92 & $3 \cdot 90$ & $7 \cdot 65$ & $16 \cdot 1$ & $52 \cdot 9 \pm 1 \cdot 1$ & $-136 \pm 4$ & $93.3 \pm 0.9$ \\
\hline$p-\mathrm{Cl}$ & 0.71 & 1.49 & $3 \cdot 05$ & $6 \cdot 49$ & $55 \cdot 3 \pm 0.9$ & $-136 \pm 3$ & $95.8 \pm 0.7$ \\
\hline$p-\mathrm{Br}$ & 0.605 & $1 \cdot 31$ & $2 \cdot 72$ & $5 \cdot 65$ & $55 \cdot 9 \pm 0 \cdot 5$ & $-135 \pm 2$ & $96 \cdot 1 \pm 0.4$ \\
\hline$p$-Me & $6 \cdot 57$ & $12 \cdot 0$ & $21 \cdot 8$ & $43 \cdot 7$ & $46 \cdot 8 \pm 1 \cdot 5$ & $-147 \pm 5$ & $90 \cdot 4 \pm 1.2$ \\
\hline$p-\operatorname{Pr}^{\mathrm{i}}$ & $5 \cdot 68$ & $10 \cdot 9$ & $20 \cdot 4$ & 39.8 & $48 \cdot 3 \pm 0 \cdot 9$ & $-143 \pm 3$ & $90.7 \pm 0.7$ \\
\hline$p$-OMe & $74 \cdot 5$ & 120 & 195 & 352 & $37 \cdot 9 \pm 1 \cdot 5$ & $-157 \pm 5$ & $84.6 \pm 1.2$ \\
\hline$m-\mathrm{Cl}$ & $0 \cdot 164$ & $0 \cdot 383$ & $0 \cdot 838$ & 1.91 & $61.5 \pm 0.8$ & $-127 \pm 3$ & $99 \cdot 3 \pm 0.6$ \\
\hline$m-\mathrm{NO}_{2}$ & 0.037 & 0.095 & $0 \cdot 222$ & $0 \cdot 521$ & $66 \cdot 6 \pm 0 \cdot 3$ & $-122 \pm 1$ & $103 \pm 0 \cdot 3$ \\
\hline$p-\mathrm{NO}_{2}$ & $0 \cdot 021$ & 0.055 & $0 \cdot 140$ & $0 \cdot 322$ & $69.3 \pm 0.4$ & $-118 \pm 1$ & $104 \pm 0 \cdot 3$ \\
\hline$m-\mathrm{Br}$ & $0 \cdot 155$ & $0 \cdot 360$ & 0.788 & 1.74 & $60 \cdot 7 \pm 0.5$ & $-130 \pm 2$ & $99.4 \pm 0.4$ \\
\hline LA & $0 \cdot 43$ & 0.92 & 1.92 & $3 \cdot 70$ & $55 \cdot 9 \pm 0 \cdot 2$ & $-141 \pm 1$ & $97 \cdot 9 \pm 0.2$ \\
\hline DMA & $0 \cdot 22$ & $0 \cdot 47$ & 0.96 & $2 \cdot 00$ & $57 \cdot 1 \pm 0.6$ & $-143 \pm 2$ & $99.6 \pm 0.5$ \\
\hline$k_{\mathrm{H}} / k_{\mathrm{D}}$ & $5 \cdot 91$ & $5 \cdot 76$ & $5 \cdot 62$ & $5 \cdot 50$ & & & \\
\hline
\end{tabular}

${ }^{\mathrm{a}}$ [oxidant] $=0.001 \mathrm{~mol} \mathrm{dm}^{-3} ;$ [hydroxy acid] $=0.5$ to $5.0 \mathrm{~mol} \mathrm{dm}^{-3}$ depending on the reactivity of the hydroxy acid

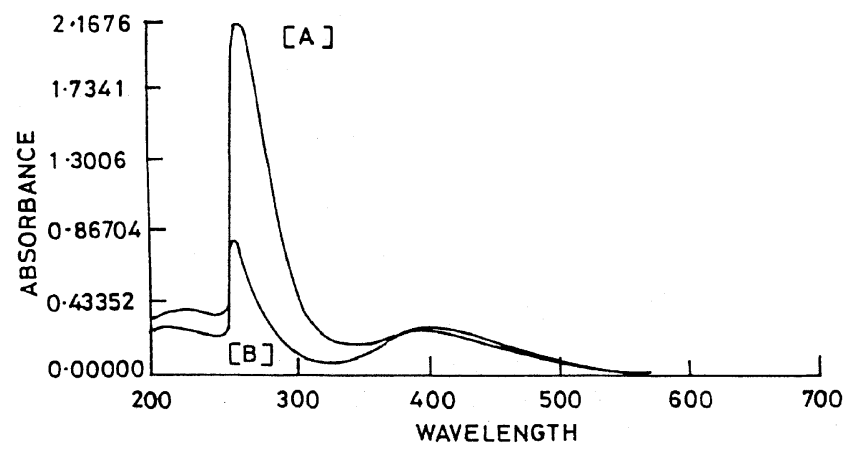

Figure 1. UV-Vis spectra of (A) $0.001 \mathrm{~mol} \mathrm{dm}^{-3}$ HABR and (B) $0.002 \mathrm{~mol} \mathrm{dm}^{-3}$ bromine; Temperature 298 K; Solvent - Glacial acetic acid.

Table 3. Temperature dependence of the reaction constant for the oxidation of substituted mandelic acids by $\mathrm{HABR}^{\mathrm{a}}$.

\begin{tabular}{lccccc}
\hline Temp. (K) & $\rho^{+}$ & $r^{2}$ & $\mathrm{sd}$ & $\psi$ & $h^{\mathrm{b}}$ \\
\hline 298 & $-2 \cdot 27 \pm 0 \cdot 01$ & 0.9999 & $0 \cdot 007$ & $0 \cdot 010$ & $-3 \cdot 89$ \\
308 & $-2 \cdot 13 \pm 0 \cdot 01$ & 0.9999 & $0 \cdot 010$ & $0 \cdot 023$ & $-3 \cdot 57$ \\
318 & $-2 \cdot 02 \pm 0 \cdot 01$ & 0.9998 & $0 \cdot 014$ & $0 \cdot 015$ & $-3 \cdot 27$ \\
328 & $-1.95 \pm 0 \cdot 01$ & 0.9998 & $0 \cdot 013$ & $0 \cdot 015$ & $-2 \cdot 96$ \\
\hline
\end{tabular}

${ }^{\mathrm{a}}$ No. of data points $=11 ;{ }^{\mathrm{b}} h=$ intercept

of the Hammett equation ${ }^{16}$ but the attempts failed to yield significant correlation (3) with Hammett's $\sigma$ values. The main deviating points correspond to para- substituents capable of electron donation by resonance viz. methoxy, methyl, isopropyl, fluoro etc. Their rates are higher than those required by their Hammett's $\sigma$ values.

$$
\log k_{2}=-2.92 \pm 0.25 \sigma-3.53
$$

$c^{2}=0.9403 ; \mathrm{sd}=0.27 ; n=11 ; \psi=0 \cdot 26 ;$ temperature $=298 \mathrm{~K}$.

Here, $n$ is the number of data points.

The rate constants were, therefore, correlated in terms of Brown's ${ }^{17} \sigma^{+}$values (4). An excellent correlation (figure 2) with large reaction constants were obtained (table 3 ):

$$
\log k_{3}=\rho^{+} \sigma^{+}+\log k_{0}
$$

Here, $k_{0}$ is rate constant for MA.

\subsection{Mechanism}

A hydrogen abstraction mechanism leading to the formation of free radicals may be discounted in view of the failure to induce polymerization of acrylonitrile $^{18}$ and the large values of the reaction constants. In most hydrogen abstraction reactions, the reaction constants have small magnitude. ${ }^{16}$

The observed substantial kinetic isotope effect $\left(k_{\mathrm{H}} / k_{\mathrm{D}}=5.91\right.$ at $\left.298 \mathrm{~K}\right)$ suggests that the $\alpha-\mathrm{C}-\mathrm{H}$ bond is cleaved in the rate-determining step. Corre- 
lation analysis of the substituent effect indicates the presence of a highly electron-deficient reaction centre in the rate-determining step. The fact that an excellent correlation is obtained with Brown's $\sigma^{+}$ values suggests that there is resonance interaction, in the transition state, between a developing positive centre at the reaction site and the substituent. Thus, the transition state approaches a carbocationic in character. Therefore, the transfer of a hydride-ion from the acid to the oxidant is indicated (scheme 1). The proposed mechanism is, however, supported by the observed negative entropy of activation for the conversion of the reactant molecules into the activated complex. As the process involves large charge separation in the transition state resulting in an increase in the total number of charges, the two ends become highly solvated. This results in an immobilization of a large number of solvent molecules, reflected in loss of entropy. Two moles of the hydroxy acids are oxidized per mole of the oxidant.

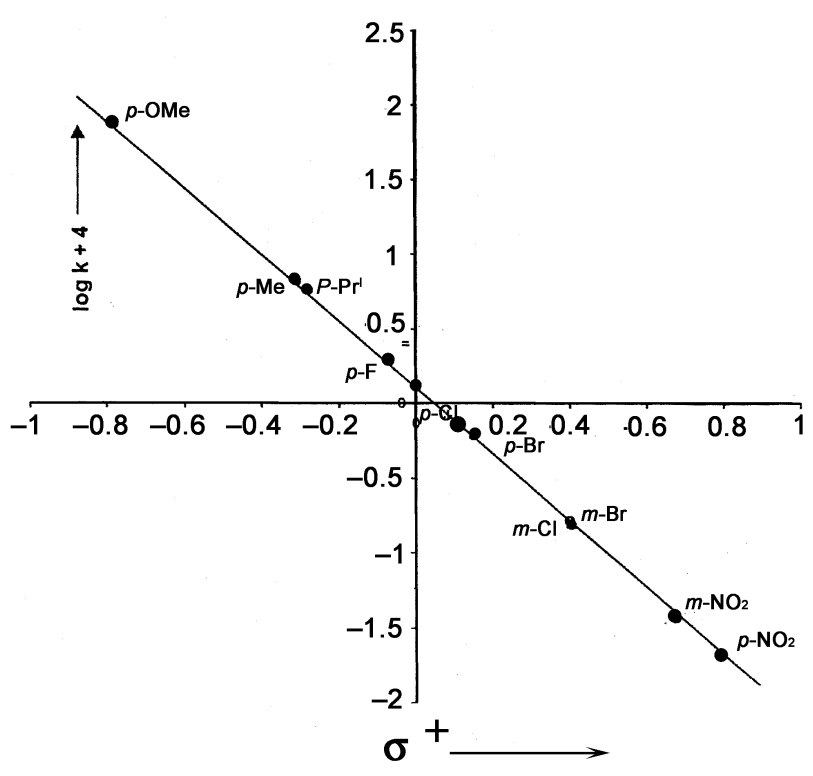

Figure 2. Brown's plot for the oxidation of MA by HABR at $298 \mathrm{~K}$.

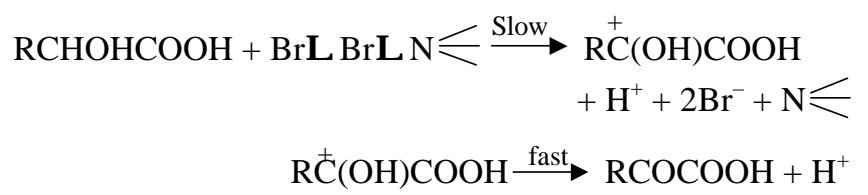

Scheme 1.
It is of interest to compare the results of oxidation of $\alpha$-hydroxy acids by other oxidants containing halogen. The rates of oxidation of $\alpha$-hydroxy acids by benzyl trimethylammonium tribromide (BTMAB) ${ }^{19}$ are reported to involve the formation of an intermediate complex in the pre-equilibrium stage, which then collapses to an acylhypobromite derivative in the rate-determining step, leading to the formation of the corresponding carbonyl compound in the fast step. However, the reactions of the oxidation of MA by both sodium N-bromobenzenesulphonamide $(\mathrm{BAB})^{20}$ and N-bromoacetamide (NBA) ${ }^{21}$ are of first order with respect to both the acid and the oxidant. The oxidation of MA by NBA is reported to involve the formation of hypobromite ester in the slow step prior to the formation of the corresponding carbonyl compound. However, the oxidation of MA by BAB undergoes oxidative decarboxylation to produce the carbonyl compound. In the oxidation by $\mathrm{PHPB}^{22}$ and BTMACI,${ }^{23}$ the reactions involve hydride-ion transfer from the acid to the oxidant to give a carbocationic species in the rate-determining step which leads to the formation of the corresponding oxyacid as the final product. This is essentially similar to the mechanism suggested in the present reaction. The oxidations of MA by two halochromates, viz. pyridinium chlorochromate $(\mathrm{PCC})^{24}$ and pyridinium bromochromate (PBC), ${ }^{25}$ are reported to involve an ester intermediate formation in the pre-equilibrium stage, which undergoes decomposition in the subsequent slow step via a cyclic, concerted and symmetrical transition state to give the corresponding oxoacid. However, the results of the correlation analysis show that the oxidation by $\mathrm{PBC}$ exhibits excellent correlation in terms of Brown's $\sigma^{+}$values, whereas PCC oxidation correlate well with Hammett's $\sigma$ values. To account for the results of the correlation analysis, an alternate mechanism involving direct hydride-ion transfer to the oxidant is also proposed. It seems that the mechanism of the oxidation of $\alpha$ hydroxy acids depends on the nature of the oxidant.

Further, it is observed that oxidations of $\alpha$-hydroxy acids by HABR follow similar reaction path to that of alcohols, ${ }^{5}$ involving hydride-ion transfer from the substrate to the oxidant in the rate-determining step. Rate laws are similar and both the oxidations exhibit substantial kinetic isotope effect of about the same order. Cleavage of the $\alpha-\mathrm{C}-\mathrm{H}$ bond is suggested in the rate-determining step of both the reactions. Oxidations of $\alpha$-hydroxy acids show excellent correlation with Brown's $\sigma^{+}$values, though the oxidation rates 
of alcohols correlate well with Charton's triparametric LDR equation. ${ }^{26}$ Regression coefficients in both the reactions are negative indicating an electrondeficient reaction centre in the transition state of the reaction.

\section{Acknowledgement}

Thanks are due to University Grants Commission (Govt. of India) for financial support.

\section{References}

1. Yavari I and Shaabani G 1994 J. Chem. Res. (S) 274; Bandgar B P, Admane S B and Jare S S 1998 J. Chem. Res. (S) 154

2. Mehta S, Kothari S and Banerji K K 2000 Int. J. Chem. Kinet. 32165

3. Choudhary K, Suri D, Kothari S and Banerji K K 2000 J. Phys. Org. Chem. 13283

4. Gangwani H, Sharma P K and Banerji K K 2000 Indian J. Chem. A39 436; Pareek A, Kothari S, Banerji K K 1996 Indian J. Chem. B35 970; Aneja M, Sharma P K and Banerji K K 2000 J. Indian Chem. Soc. 77294

5. Goyal and Kothari S 2002 J. Chem. Res. (S) 2; (M) 1259

6. Banerji K K 1978 J. Chem. Res. (S) 193; (M) 2561

7. Levesley P and Waters W A 1955 J. Chem. Soc. 217

8. Vogel A I 1956 A text book of practical organic chemistry (London: Longmans Green) p. 774
9. Kemp T J and Waters W A 1964 J. Chem. Soc. 1194

10. Orton K J P and Bradfield A J 1924 J. Chem. Soc. 960; Orton K J P and Bradfield A J 1927 J. Chem. Soc. 983

11. Feigl F 1966 Spot tests in organic analysis (Amsterdam: Elsevier) p. 485

12. Pollock J R A and Stevans R (eds) 1964 Dictionary of organic compounds (London: Eyre and Spottiswoode) vol. 1, p. 359

13. Exner O 1966 Collect. Czech. Chem. Commun. 31 3222

14. Exner O 1973 Prog. Phys. Org. Chem. 10411

15. Pink J M and Stewart R 1971 Can. J. Chem. 49654

16. Wiberg K B 1963 Physical organic chemistry (New York: John Wiley)

17. Brown H C and Okamoto Y $1958 \mathrm{~J}$. Am. Chem. Soc. 804979

18. Mehnert R and Brede O 1984 Radiat. Phys. Chem. 23463

19. Goyal S, Kothari S and Banerji K K 1996 Indian J. Chem. B35 1180

20. Mathur A, Sharma V and Banerji K K 1988 Indian J. Chem. A27 123

21. Bishnoi M L, Negi S C and Banerji K K 1986 Indian J. Chem. A25 660

22. Aparna P, Kothari S and Banerji K K 1993 Indian J. Chem. A32 1086

23. Goyal A and Kothari S 2003 Bull. Chem. Soc. Jpn. 761

24. Banerji K K 1978 J. Chem. Res. (S) 193 (M) 2561

25. Aparna P, Kothari S and Banerji K K 1994 J. Chem. Res.(S) 367; (M) 2123

26. Charton M and Charton B 1988 Bull. Soc. Chim. Fr. 199, and references cited therein 